\title{
Gestational diabetes and fetal outcome: a study in a tertiary care centre
}

\author{
V. G. Vanamala*
}

Department of Obstetrics and Gynecology, VRK Womens Medical College and Hospital and Research Centre, Aziz Nagar, Rangareddy, Hyderabad, India

Received: 24 July 2017

Accepted: 22 August 2017

\section{*Correspondence:}

Dr. V. G. Vanamala,

E-mail:vgvanamala@gmail.com

Copyright: $\odot$ the author(s), publisher and licensee Medip Academy. This is an open-access article distributed under the terms of the Creative Commons Attribution Non-Commercial License, which permits unrestricted non-commercial use, distribution, and reproduction in any medium, provided the original work is properly cited.

\begin{abstract}
Background: Due to urbanization and sedentary lifestyle, dietary changes, and increased obesity of the people, the incidence of GDM is steadily on the rise. It is associated with severe morbidity to the mother and the child. It is therefore imperative that an early diagnosis needs to be done so that appropriate treatment can be given.

Methods: 1654 women who were included in the study were in their $24-28$ weeks of gestation. A standardized questionnaire was formatted and details regarding the age, weight, body mass index (BMI), parity, previous medical and obstetrics history and familial history of diabetes, tests for glucose levels, complete blood picture, routine urine examination. Oral glucose tolerance test was done for all the patients after fasting overnight.

Results: 87 (5.3\%) of them were positive for OGTT and were considered to have Gestational Diabetes mellitus. $67.8 \%$ of the patients were in the $25-30$ age group. $41.4 \%$ were pregnant for the first time and $58.6 \%$ were multi gravid. The majority of the patients had a BMI between 26-30. Most of the babies had a birth weight of above $3 \mathrm{kgs}$. Out of them, $39(44.8 \%)$ had a birth weight between 3.1-3.5kgs. <2kgs were seen in $7(8.0 \%)$ patients.

Conclusions: GDM complicates pregnancy and results in higher frequency of adverse effects in the mother and new born. Thereby, early detection can result in prompt treatment and lowering the morbidity of the fetal outcomes.
\end{abstract}

Keywords: Fetal outcomes, Gestational diabetes, Oral glucose tolerance test, Pregnant mothers

\section{INTRODUCTION}

Pregnancy causes a few changes to occur in the carbohydrate metabolism in the mother. Along with the advancement in the state of pregnancy, insulin resistance and diabetogenic stress due to placental hormones take place, resulting in the increase in insulin secretion. An inadequate compensation of insulin, if inadequate, may result in the mother having a higher sugar level in the blood. This elevated glucose level or glucose intolerance during pregnancy is called gestational diabetes (GDM). ${ }^{1}$ Due to urbanization and sedentary lifestyle, dietary changes, and increased obesity of the people, the incidence of GDM is steadily on the rise. The prevalence of GDM varies in different regions. Overall the incidence of GDM of estimated to be 7\%, with an annual increase of 200,000 cases. In the US alone, the GDM is reported to be $14 \%$ of all the pregnancies. ${ }^{2}$ Amongst the Koreans, the incidence is 2-5\%. ${ }^{3}$ In Europe, America and Asia, in general, the prevalence is reported to be $3-6 \% .^{4-6}$

In comparison to the white women the Indian women are prone to GDM eleven fold, eight fold in South East Asia, and three fold in Arab and Afro- Caribbean countries. ${ }^{7}$ It is known to cause complications in $2-3 \%$ of all the pregnancies. It is also associated with a higher incidence of diabetes to the mother later on in life. ${ }^{8}$ The major morbidities seen in the new born is respiratory distress, 
hypocalcemia, polycythemia, hypoglycemia, growth restriction, hypomagnesemia, and congenital malformations. A $42.9 \%$ mortality in the infants with mothers with poor glycaemic control was also reported. ${ }^{9}$

It is therefore imperative that an early diagnosis needs to be done so that appropriate treatment can be given.

\section{METHODS}

This observational study was conducted by the Department of Gynecology at VRK Women's Medical College over a period of three years from July-2014 to Mach -2017. 1654 patients who had come to the antenatal clinic of our hospital were screened for gestational diabetes mellitus (GDM). All the women who were included in the study were in their $24-28$ weeks of gestation. Patients with a history of diabetes prior to the present study were excluded. Other criteria for exclusion of the patients included carcinoma, tuberculosis, congestive heart failure, renal failure and other major illnesses.

The study procedure was explained clearly to the patients and informed consent was taken from all of them.

A standardized questionnaire was formatted and details regarding the age, weight, body mass index (BMI), parity, previous medical and obstetrics history and familial history of diabetes details like age, weight, body mass index (BMI), parity, medical history, previous history and familial history of diabetes, obstetric history were taken followed by complete physical examination, tests for glucose levels, complete blood picture, routine urine examination. Oral glucose tolerance test was done for all the patients after fasting overnight.

Oral glucose tolerance test (OGTT) was done after an overnight fast. $75 \mathrm{~g}$ of pure glucose was mixed with $100 \mathrm{ml}$ of water and the patient was made to drink this mixture. $2 \mathrm{ml}$ of blood was collected in a sodium fluorideoxalate bottle immediately. This was considered as 0 hour time. Blood samples were collected again at 1,2 and 3 hours respectively. Plasma glucose levels were interpreted using NDDG criteria.

\section{RESULTS}

A total of 1654 pregnant women attending the outpatient and the inpatient wards of our hospital had satisfied the inclusion criteria and were included in the study.

Out of them 96 patients had abnormal fasting ad post parandial blood sugar levels and were tested for OGTT.

$87(5.3 \%)$ of them were positive for OGTT and were considered to have Gestational Diabetes mellitus (GDM) (Figure 1).

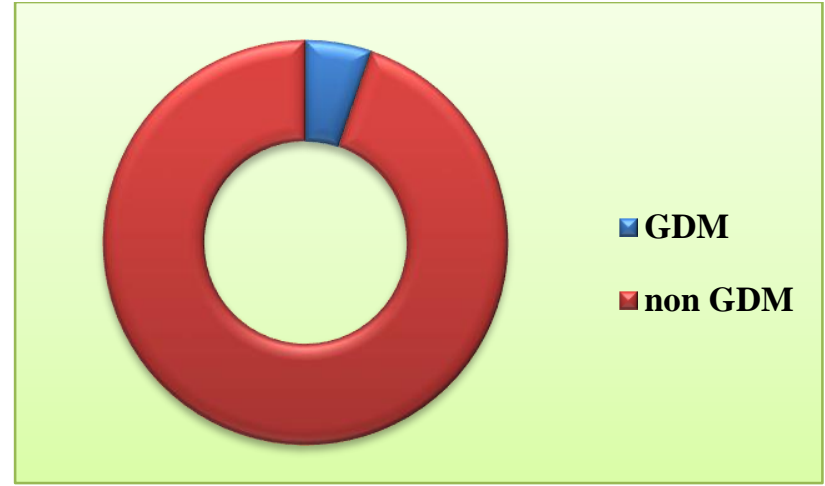

Table 1: Prevalence of GDM.

Most of the patients were in the 25-30 age group (67.8\%). Around $12 \%$ of them were below the age of 25 years. 36 (41.4\%) were pregnant for the first time and 51 (58.6\%) were multi gravid. 23 patients $(26.4 \%)$ had a BMI of $18-$ 24 , while $22(25.3 \%)$ has an Index of $>30$. However, the majority of the patients had a BMI between 26-30 (Table $1)$.

Table 1: Demographic details.

\begin{tabular}{|lll|}
\hline Details & Number & $\%$ \\
\hline Age & & \\
\hline$<25$ & 11 & 12.6 \\
\hline $25-30$ & 59 & 67.8 \\
\hline$>30$ & 17 & 19.5 \\
\hline Gravida & & \\
\hline Primi & 36 & 41.4 \\
\hline Multi & 51 & 58.6 \\
\hline BMI & & \\
\hline$<18$ & 6 & 18.4 \\
\hline $18-24$ & 23 & 26.4 \\
\hline $24-30$ & 36 & 41.4 \\
\hline$>30$ & 22 & 25.3 \\
\hline
\end{tabular}

The most predominant mode of delivery of the women was spontaneous (35.6\%). 26 patients $(29.9 \%)$ had opted for elective surgery. Emergency procedure was performed for 18 patients $(20.7 \%$ ) (Figure 2).

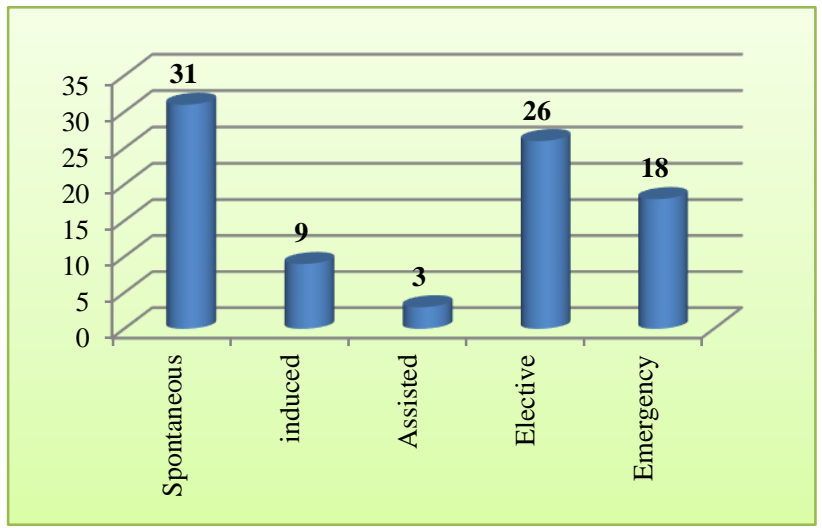

Figure 2: Mode of delivery. 
Most of the babies had a birth weight of above $3 \mathrm{kgs}$. Out of them, $39(44.8 \%)$ had a birth weight between 3.1 $3.5 \mathrm{kgs}$. $<2 \mathrm{kgs}$ were seen in $7(8.0 \%)$ patients (Figure 3 ).

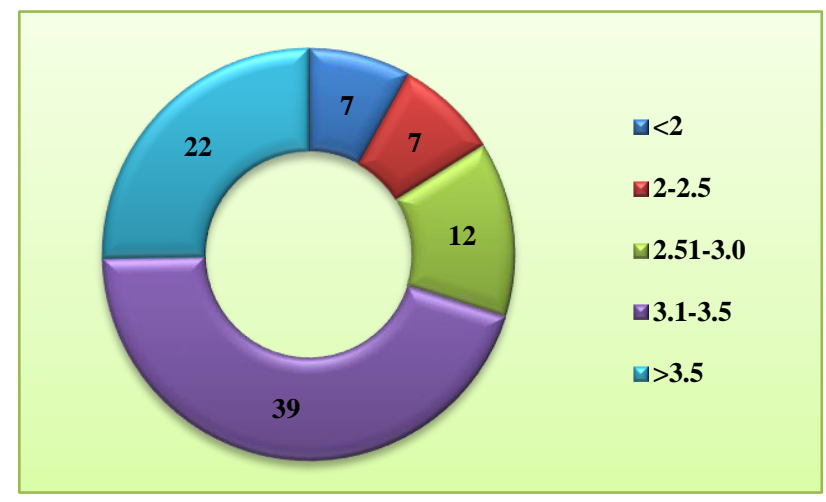

Figure 3: Birth weight of the infant.

Macrosomia was the most common complications among the neonates $(29.9 \%)$. Respiratory distress was observed in 6 patients $(6.9 \%)$. Other complications that were observed were hypoglycemia, hypocalemia and jaundice. Congenital abnormality was seen in 2 (2.3\%) (Figure 4).

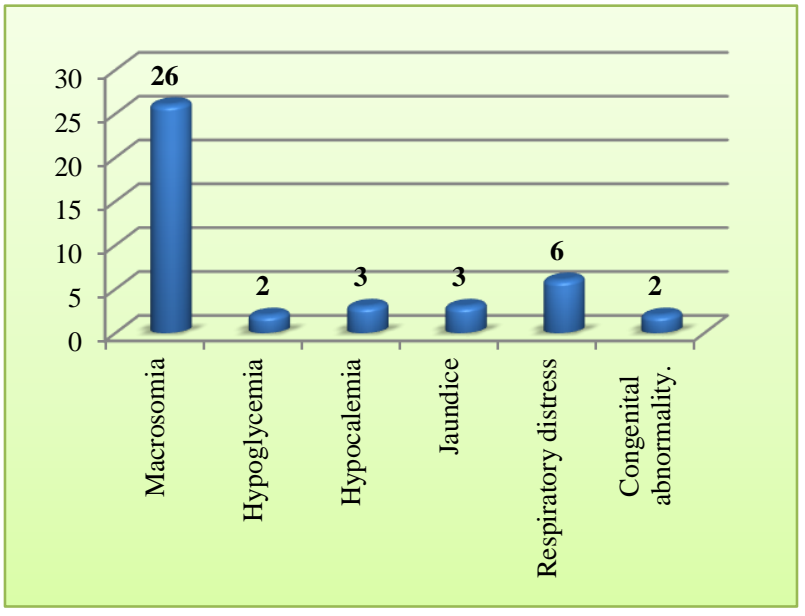

Figure 4: Fetal complications.

\section{DISCUSSION}

Glucose intolerance or Diabetes mellitus, which is diagnosed for the first time during pregnancy is called gestational diabetes mellitus. It appears usually during pregnancy, but disappears soon after, probably due to the increased hormonal changes during this period. Normally, the presence of GDM is due to the impaired insulin resistance or reduced glucose utilization due to a maternal defect, thereby ensuring the reduced insulin secretory capacity of the woman. This may lead to at a later stage, full blown diabetes in the woman. Often, it is a result of risk factor such as overweight, advanced age and previous history of GDM. ${ }^{10-12}$

The prevalence of GDM is estimated to be between 1$14 \%$ worldwide. The present study has shown a prevalence of $5.3 \%$ of GDM in women in our area. A similar prevalence of $8.6 \%$ mothers was seen in a study by Malak, in Kingdom of Saudi Arabia. ${ }^{13} 4.2 \%$ incidence was reported in a study by Dudhwadkar and Fonseca in Mumbai. ${ }^{14}$ In other similar studies, such as Nilofer in Devangere, Karnataka, a rate of $6 \%$ and Wahi et al from Jammu with a rate of $6.94 \%$ were identified. ${ }^{15,16}$

The most common age group affected observed in our study was 25-30 years, which was far higher than patients below 25 years of a ge. A similar result was observed by Rajput et al, who reported an increase of 3.8 times more in a women of greater than 25 years rather than lesser. ${ }^{17}$ A similar observation was made by Seshaiah et al in their study. ${ }^{18}$ Around $56 \%$ of the mothers with GDM in a study by Dudhwadkar and Fonseca were between the age group 26-30, further corroborating our results.

The BMI of most of the patients in our study was between 24-30. A positive association was observed in the present study berween obesity and GDM. This was in accordance to many other studies. ${ }^{18-21}$ Primi gravida was seen in $41,1 \%$ of the patients, while it was observed that on increase in the parity, the risk of GDM increases. Similar reports were observed by Dudhwadkar et al and Rajput et al. ${ }^{14,17}$

$35.6 \%$ of the patients in our study had normal vaginal delivery, while $29.9 \%$ had elective surgery. Most if the patients who went in for caesarian section had babies with macrosomia. The incidence of macrosomia was the predomiant complication among the neonates, with mother having GDM. A similar result was reported by Odar et al, wherein the number of vaginal deliveries and elective surgeries were similar though the number of caesarian sections were indicated more in the case of macrosomia babies. ${ }^{22}$ However, in a few studies, there were increase of the caesarian sections compared to normal deliveries..$^{23,24}$

\section{CONCLUSION}

Multigravida women with a history of GDM in their earlier pregnancies should be extra careful during the consequent pregnancies. A simple blood test is useful for the detection of GDM and early treatment of the disease. This restricts the morbidity and mortality of the child as well as the mother thereby lowering the hospitalization cost. Women in their first pregnancy with obesity also need to take appropriate preventive measures.

\section{Funding: No funding sources}

Conflict of interest: None declared

Ethical approval: The study was approved by the Institutional Ethics Committee

\section{REFERENCES}

1. Seshiah V, Das AK, Balaji V, Joshi SR, Parikh MN, Gupta S. Diabetes in pregnancy study group. 
gestational diabetes mellitus-guidelines. J Assoc Physicians India. 2006;54:622-8.

2. Kim C, Newton KM, Knopp RH. Gestational diabetes and the incidence of type 2 diabetes: a systematic review. Diabetes Care. 2002;25:1862-8.

3. Jang HC, Cho YM, Park KS, Kim SY, Lee HK, Kim MY, Yang JH, Shin SM. Pregnancy outcome in Korean women with gestational diabetes mellitus diagnosed by the Carpenter-Coustan criteria. J Korean DiabAssoc. 2004;28:122-130.

4. Ahkter J, Qureshi R, Rahim F, Moosvi S, Rehman A, Jabbar A et al. Diabetes in pregnancy in Pakistani women: prevalence and complications in an indigenous South Asian community. Diab Med. 1996;13(3):189-191.

5. Bancrofti K, Tuffnel GC, Mason GC, Rogerson LJ, Mansfield M. A randomised controlled pilot study for the management of gestational impaired glucose tolerance. BJOG. 2000;107:959-963.

6. Young C, Kuehl TJ, Sulak PJ, Allen RS. GDM screening in subsequent pregnancy of previously healthy patients. Am J Obstet Gynecol. 2000;181(4):798-802

7. Dornhost A, Pateson EM, Nicholls JSD, et al. High Prevalence of GDM in women from ethnic minority groups. Diab Med. 1992;9:820-5.

8. Davey RX, Hamblin PS. Selective versus universal screening for gestational diabetes mellitus: An evaluation of predictive risk factors. Med J Aust. 2001;174:118-21.

9. Otolorin EO, Famuyiwa OO, Bella AF, Dawodu AH, Adelusi B. Reproductive performance following active management of diabetic pregnancies at the university college hospital, Ibadan, Nigeria. Afr J Med Med Sci. 1985;14:155-60.

10. King H. Epidemiology of glucose intolerance and gestational diabetes in women of child bearing age. Diabetes Care. 1998;21(Suppl 2):9-13.

11. Engelgau MM, Herman WH, Smith PJ, German RR, Aubert RE. The epidemiology of diabetes and pregnancy in the US, 1988. Diab Care. 1995;18(7):1029-33.

12. Shefali AK. Pregnancy outcomes in pre-gestational and gestational diabetic women in comparison to non-diabetic women-a prospective study in asian indian mothers. J Assoc Physicians India. 2006;54:613-8.

13. Al-Hakeem MM. Pregnancy outcome of gestational diabetic mothers: experience in a tertiary center. J Fam Community Med. 2006 May-Aug;13(2):55-59.
14. Dudhwadkar AR, Fonseca MN. Maternal and fetal outcome in gestational diabetes mellitus. Int $\mathbf{J}$ Reprod Contracept Obstet Gynecol. 2016;5:3317-21.

15. Nilofer AR, Raju VS, Dakshayini BR, Zaki SA. Screening in high-risk group of gestational diabetes mellitus with its maternal and fetal outcomes. Indian J Endocrinol Metab. 2012;16:74-8.

16. Wahi P, Dogra V, Jandial K, Bhagat R, Gupta R, Gupta $S$ et al. Prevalence of gestational diabetes mellitus and its outcomes in Jammu region. J Assoc Physicians India. 2011;59:227-30.

17. Rajput R, Yadav Y, Nanda S, Rajput M. Prevalence of gestational diabetes mellitus and associated risk factors at a tertiary care hospital in Haryana. The Indian J Med Res. 2013 Apr;137(4):728.

18. Seshiah V, Balaji V, Balaji MS, Paneerselvam A, Arthi $T$, Thamizharasi $M$ et al. Prevalence of gestational diabetes mellitus in South India (Tamil Nadu): a community based study. J Assoc Physicians India. 2008;56:329-33.

19. Soheilykhah S, Mogibian M, Rahimi-Saghand S, Rashidi M, Soheilykhah S, Piroz M. Incidence of gestational diabetes mellitus in pregnant women. International J Reprod BioMed. 2010;8(1):24-8.

20. Sreekanthan K, Belicita A, Rajendran K, Vijayakumar A. Prevalence of Gestational diabetes mellitus in a medical college in South India: a pilot study. Indian J Clin Pract, 2014;25(4).

21. Swami SR, Mehetre R, Shivane V, Bandgar TR, Menon PS, 11. Shah NS. Prevalence of carbohydrate intolerance of varying degrees in pregnant females in western India (Maharashtra): A hospital-based study. J Indian Med Assoc. 2008;106:712-4.

22. Odar E, Wandabwa J, Kiondo P. Maternal and fetal outcome of gestational diabetes mellitus in Mulago Hospital, Uganda. Afr Health Sci. 2004;4(1):9-14.

23. Naylor CD, Sermer M, Chen E, Sykora K. Caesarian delivery in relation to birth weight and glucose gestational tolerance. JAMA. 1996;275(15):116470 .

24. Coustan D. Management of GDM: a self-fulfiling prophecy? JAMA. 1996;275:895-900.

Cite this article as: Vanamala VG. Gestational diabetes and fetal outcome: a study in a tertiary care centre. Int J Reprod Contracept Obstet Gynecol 2017;6:4458-61. 